\title{
GESUNDHEITSFONDS
}

\section{Auswirkungen auf Leistungserbringer absehbar}

\section{Clarissa Kurscheid und Frank Schulz-Nieswandt}

\begin{abstract}
Der Gesundheitsfonds soll den Wettbewerb unter den Krankenkassen der Gesetzlichen Krankenversicherung (GKV) verstärken. Dabei wurde mit dem Fonds ein Hybrid entwickelt, welches beiden großen politischen Parteien die Möglichkeiten offen lässt, ihr favorisiertes Konzept zur sozialen Absicherung durchzusetzen. Die Bündelung der Finanzströme hat erhebliche Auswirkungen auf die Leistungserbringer, weniger durch den Gesundheitsfonds selbst als vielmehr durch die damit verbundenen weiteren Veränderungen in der Finanzierungs- und Vertragsordnung des GKVSystems.
\end{abstract}

Mit dem GKV-Wettbewerbsstärkungsgesetz zum 1. April 2007 wurde mit der Wirkung ab Januar 2009 der Gesundheitsfonds zur Bündelung der Finanzströme eingesetzt. In der Betrachtung von möglichen Finanzausgleichssystemen zwischen Versicherungen und ihren Versicherten kann zwischen internen und externen Finanzausgleichssystemen unterschieden werden. Erstere zeichnen sich dadurch aus, dass ausschließlich ein interner Austausch von Zahlungsströmen stattfindet - hier als Risikostrukturausgleich (RSA) zwischen den Krankenkassen. Dieses Verfahren wird bis dato angewandt.

Ab dem 1. Januar 2009 wird das Verfahren extern ablaufen. Die Veränderung liegt darin, dass die Versicherten ihren Beitrag direkt in den Fonds bezahlen (1) und dieser dann eine Transferzahlung an die Kasse vornimmt. Institutionell wird also ein intermediäres Finanzgebilde in den Gesamtmechanismus eingezogen. Jede Krankenkasse erhält folglich einen einkommensunabhängigen Pauschalbetrag und weitere $\mathrm{Zu}$ - oder Abschläge, die sich nach Alter, Geschlecht und Morbidität orientieren. (2)

Die Zulage über die Grundpauschale hinaus soll die unterschiedliche Versichertenstruktur ausgleichen. Hinzukommen kassenspezifische Ausgleiche oder Vergünstigungen, die je nach Wirtschaftslage von einer Krankenkasse

\footnotetext{
Anmerkungen

(1) Um weiteren bürokratischen Aufwand zu vermeiden, werden die Beiträge nach wie vor an die Kasse gezahlt und dann aber sofort weitergeleitet.

(2) Der Ausgleich erfolgt über eine Neustrukturierung des Risikostrukturausgleiches. Hierbei werden 80 besonders teure Krankheiten gesondert berücksichtigt.
}

erhoben oder erstattet werden können. Sogenannte »versicherungsfremde Leistungen « (etwa der »Familienlastenausgleich « in der Gesetzlichen Krankenversicherung) werden ab 2009 aus Steuermitteln finanziert. Morphologisch bleibt hier das Problem, dass der Konzeptbegriff der Versicherungsfremdheit eine bewusste politische Abwertung der solidarischen Umverteilungsvorgänge innerhalb der Sozialversicherung bedeutet. Aus der Sicht einer sinnadäquaten Betriebsmorphologie der Gesetzlichen Krankenversicherung als solidarische Sozialversicherung müsste sogar die Orientierung an der Privatversicherung als »fremd $«$ bezeichnet werden.

Die Steuermittel sollen jährlich gesteigert werden, bis sie 2014 einen Betrag von insgesamt 14 Milliarden Euro erreichen. Dieser Betrag würde dann circa zehn Prozent der jetzigen Ausgaben der Gesetzlichen Krankenversicherung ausmachen. Der Beitragssatz soll in Zukunft nicht mehr durch die Kassen, sondern von der Bundesregierung festgelegt werden. Dieser Verordnungsweg hat sein Vorbild in der gesetzlichen Regelung des SGB XI.

Diese Veränderungen werden wohl erhebliche Auswirkungen auf den Kassenwettbewerb haben. Zunächst ist die finanzielle Handlungsoption des Zusatzbeitrages ein unter Umständen nur eingeschränktes Mittel, notwendige Finanzbedarfe zu realisieren, insbesondere wenn der überwiegende Anteil der Mitglieder über niedrige Einkommen verfügt. Kassen, deren Mitglieder bessere Einkommensstrukturen vorweisen, bieten sich demnach größere Handlungsspielräume. Wechselanreize für Versicherte werden sich entsprechend stark aus den möglichen Zusatzbeiträgen ergeben. Aus Sicht einer Solidarlogik kann es also, betrachtet man wiederum die Problematik aus der Perspektive einer sinnadäquaten Betriebsmorphologie der Gesetzlichen Krankenversicherung als Sozialversicherung, adverse Anreizeffekte geben, die zu unerwünschten Risikoselektionen führen können. Die Gefahr besteht, dass hier - trotz der Evolution von Qualitätsverbesserungen durch neue innovative Versorgungsformen ( $u$. a. die Integrationsversorgung nach § 140a-d SGB V) und evidenzgestützter Patientenpfade (Qualitätswettbewerb) mit diesen kassenindividuellen Beitragsanhebungen zur Sicherung der Finanzlage als Abweichungen vom Einheitsbeitragssatz ein Preiswettbewerb einsetzt, der entweder Kassen zu Grenzanbietern am Kassenmarkt werden lässt, Konkurse herbeiführt oder zu weiteren Fusionen und zum Betriebsgrößenwachstum zwingt oder negative Risikoselektionen im Versichertenmarkt erzwingt. Dabei ist wiederum zu bedenken, dass der bisherige Risikostrukturausgleich (trotz seiner Unvollkommenheiten) dazu 


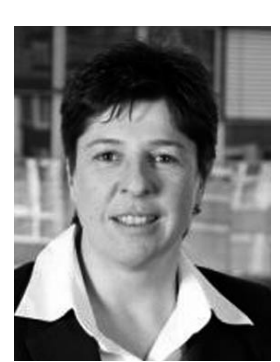

Dr. Clarissa Kurscheid studierte nach einer Ausbildung und praktischer Tätigkeit als Physiotherapeutin Betriebswirtschaftslehre und Gesundheitsökonomie an der Universität Köln, an der sie auch 2005 promovierte. Sie arbeitet seit 2003 am Seminar für Sozialpolitik und lehrt dort sowie an verschiedenen Fachhochschulen u. a. Europa Fachhochschule Fresenius in Köln und Katholischen Fachhochschule Freiburg. Ihre Arbeits- und Forschungsschwerpunkte liegen im Bereich des Gesundheitswesens, der Versorgungsforschung, alternative Versorgungsformen, Integrationsversorgung, Organisation von Gesundheitsbetrieben sowie Kooperationen und Konflikte in Organisationen. Neben der Tätigkeit als Dozentin ist sie als Beraterin für Projekte mit integrativem Versorgungsansatz aktiv. Hierbei ist sie sowohl wissenschaftlich begleitend tätig als auch im konzeptionellen und operativen Bereich. E-Mail kurscheid@wiso.uni-koeln.de

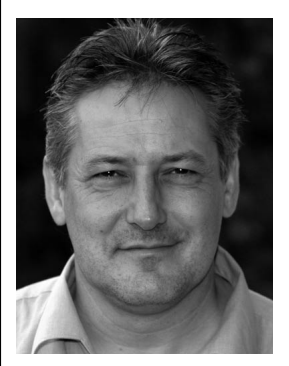

Prof. Dr. Frank Schulz-Nieswandt ist Direktor des Seminars für Sozialpolitik und des Seminars für Genossenschaftswesen der Universität zu Köln und dort auch Dekan der Wirtschafts- und Sozialwissenschaftlichen Fakultät. Er ist Vorsitzender der Gesellschaft für Sozialen Fortschritt, Vorsitzender des Wissenschaftlichen Beirates des Bundesverbandes öffentliche Dienstleitungen (BVÖD), Mitglied und Sprecher des Wissenschaftlichen Beirates des Deutschen Zentrums für Altershilfe in Berlin, Mitglied des Vorstandes des Deutschen Vereins für öffentliche und private Fürsorge und Mitglied des Vorstandes von Ciriec an der Universität Lüttich. Seine Forschungsschwerpunkte sind Anthropologie und Kulturgeschichte der Sozialpolitik und solidarischer Selbsthilfegebilde, das Gesundheitswesen im Lichte gerontologischer Forschungen, das Europarecht, europäische Sozialpolitik und öffentliches Wirtschaften.

E-Mail schulz-nieswandt@wiso.uni-koeln.de

diente, kassenseitige Versichertenselektionen zu minimieren, da das spezifische institutionelle Setting der Kassenwahlfreiheit und des Kontrahierungszwanges der Kassen gerade diese ökonomisch rationalen, sozialversicherungsmorphologisch aber unerwünschten betriebspolitischen Reaktionsmuster plausibel werden lässt.

So laufen Preiswettbewerb und Qualitätswettbewerb unvermittelt - desintegriert - nebeneinander her. Es kann sogar zum kumulativen Zirkel kommen: Fiskalisch begünstigte Kassen mit guter Risikostruktur gewinnen zunehmend qualitätsbezogene Wettbewerbsvorteile. Umgekehrt bei fiskalisch schwachen Kassen. Das Gesundheitswesen ist damit weit von einem integrierten Preis-
Qualitäts-Wettbewerb entfernt. Es wird mitunter von der Neuregelung des Risikostrukturausgleichs abhängen, inwieweit hier die Erosionen der Kassenlandschaft und die Risikoselektionszwänge der Kassen im Versichertenmarkt kompensiert werden.

Genau diese Gefahr wird potenziert durch das weitere neu eingeführte Instrument der Wahltarife, die als monetäre (Prämienzahlungen o. Ä.) oder versorgungsbezogene Wahltarife (Therapierichtungen), den Kassen als Wettbewerbsparameter angeboten werden. Die Problemkette ist jetzt vom anderen Ende her zu denken: Die Möglichkeit von versorgungsbezogenen Wahltarifen bietet für die Kassen eine Form des Qualitätswettbewerbs.

Dieses Instrument der Wahltarife hat nun allerdings explizit Relevanz für die Leistungsanbieter. Unter versorgungsbezogenen Wahltarifen ist insbesondere die Teilnahme an innovativen und integrierten Versorgungsformen gemeint. Diese können zwischen Kassen und Leistungserbringer mittels selektivvertraglicher Vereinbarung abgeschlossen werden. Die gesetzlichen Grundlagen hierfür sind $\mathrm{u}$. a. Modelle zur integrierten Versorgung (§ 140a SGB V), hausarztzentrierte Versorgung (§ 73b SGB V), besondere ärztliche Behandlungsformen ( $\$$ 73c SGB V), Modellvorhaben ( 63 SGB V) und strukturierte Behandlungsprogramme bei chronischen Erkrankungen (§ 137 f. SGB V). Auch wenn der Vorteil für einzelne Leistungserbringer abhängig ist von den jeweiligen Versorgungsverträgen, bietet sich dennoch hier ein finanzieller Anreiz, zusätzliche Versorgungsverträge mit Einzelkassen abzuschließen (vgl. Gerlinger, Mosebach \& Schmucker, 2007:24). Mit diesen zusätzlichen Versorgungsverträgen kann die derzeitige sektorale Fragmentierung gemindert werden und typische Schnittstellenprobleme finden größere Beachtung (Schulz-Nieswandt \& Kurscheid 2004).

Neue Vertragsformen gehen für die Leistungserbringer und Akteure mit neuen Betriebsformen einher (Kurscheid, 2007:35). Folglich werden durch die veränderten gesetzlichen Regelungen »Experimentierfelder « eröffnet und Entdeckungsprozesse angestoßen (Hartweg, 2007). Dabei wird entscheidend sein, dass eine Anreizneutralität unabhängig von der Organisationsform für die Leistungserbringer gewährleistet ist. Die Leistungserbringer können in verschiedenen Organisationsformen als Vertragspartner auftreten und die Vertragsbeziehung kann über die Steuerung von Versorgungsprozessen bis zur Übernahme von Budgetverantwortung gehen (Braun \& Güssow, 2006: 68). Das bedeutet auch, dass künftige monetäre Zuschläge der Kassen für die Versicherten nicht an eine Organisationsform gebunden sind. Konsequenterweise können potenzielle Partner von Krankenkassen ihre Rechtsform frei wählen. So wird es möglich sein, auch als Managementgesellschaft eines Leistungserbringer-Netzwerkes oder Medizinisches Versorgungszentrum, mit den Kassen Verträge auszuhandeln.

Mit der gesetzlichen Öffnung im Hinblick auf neue Betriebsformen bedarf es aber verstärkter Bemühungen zum Wandel für die Akteure in den Versorgungslandschaften. Zunächst werden für die Umsetzung möglicher Konzepte von neuen Versorgungsformen eine neue Generation von Betrieben und Netzwerken benötigt. In der interprofessionellen Sichtweise steht bei Netzwerken berufsgruppenund fachübergreifende Zusammenarbeit als notwendige 
Bedingung im Vordergrund. Eine in diesem Zusammenhang häufig geäußerte Befürchtung, dass mit der Einführung von Unternehmensnetzwerken die rechtliche Eigenständigkeit sowie die eigene personale Identität der Professionen verloren geht, trifft nicht zu. Eine wesentliche Voraussetzung allerdings ist eine geforderte Durchlässigkeit innerhalb der Organisationsgrenzen, damit ein $\mathrm{Zu}$ und Abfluss von Informationen gewährleistet ist (Kurscheid, 2007:41). Dabei bedarf es für die aktive Umsetzung und den gewandelten Umgang mit den Patienten, mit Aufbau- und Ablauforganisationen im medizinischen System, eine neue inter- und auch intraprofessionelle Kommunikationskultur.

Unter Berücksichtigung der enormen Schnittstellenprobleme und der sektoralen Fragmentierung im Gesundheitswesen werden künftig möglicherweise diejenigen Akteure für die Kassen von Interesse sein, die in der Lage sind, mit ihren Versorgungsansätzen schnittstellenübergreifend aktiv zu sein. Dabei wird es auch darum gehen, den Informationsfluss zwischen den einzelnen Leistungserbringern zu steuern und zu fördern. Diese Aufgabe obliegt dann auch nicht mehr ausschließlich medizinisch ausgebildeten Akteuren, sondern hier sind vorwiegend Akteure mit medizinisch übergreifenden Kenntnissen und organisationalen Kompetenzen gefragt. Ein großer Vorteil dieser verhältnismäßig neuen Entwicklung könnte mit der Überwindung von Schnittstellenproblemen in einer möglichen Verminderung von Parallelstrukturen und Doppeluntersuchungen liegen. Potenzielle Akteure für diese neuen Tätigkeiten können Case Manager und Care Manager sein. Inwieweit die genannten Akteure den Wandel gestalten, obliegt ihrer persönlichen Berufssozialisation und ihrer persönlichen Offenheit für neue Perspektiven (Blonski, 2007:30).

\section{Fazit}

Es handelt sich beim Gesundheitsfonds um eine Frage der Systemfinanzierung. Bei »vogelflugartiger « Betrachtung des Gesundheitswesens geht es um systematische Fragen der Außenfinanzierung. Anbieterrelevant sind dagegen die Innenfinanzierungsströme, also vergütungsund honorierungsökonomische Steuerungen. Hier geht es um Effizienz- und Effektivitätsfragen, letztendlich um die Orientierung an politisch gewollten Outcomes.

Der Druck auf eine Reform der Systemfinanzierung resultiert weitgehend aus der Arbeitsmarktabhängigkeit der bruttolohnbezogenen Beitragsbemessung im Fall von sozialversicherungspflichtiger abhängiger Beschäftigung. Die Definition von Ersatzeinkommen, die Frage von alternativen (haushaltseinkommensbezogenen) Beitragsbemessungsgrundlagen, von Beitragsbemessungs- und Pflichtversicherungsgrenzen (damit auch die Frage des dualen Systems von Gesetzlicher und Privater Krankenkasse tangierend) usw. resultieren erst aus dieser Problematik einer mangelnden Nachhaltigkeit arbeitslosigkeits-, aber auch demografieabhängiger Umlagefinanzierungstechnik. Wachstumsabhängig, auch konjunkturanfällig, sind natürlich auch steuerfinanzierte Systeme.

Die Ausgliederung einzelner Umverteilungskomponenten aus der beitragsfinanzierten Logik in den öffentlichen
Steuerhaushalt wird die Morphologie der Gesetzlichen Krankenversicherung verändern. Die Frage ist, ob sich die Ausgleichsverpflichtung dauerhaft sichern lässt. Die Sozialversicherungsbeiträge waren zumindest zweckgebunden. Die Konkurrenz der Verwendungszwecke im Steuerhaushalt ist intensiver. Die Umverteilungsdynamik im bisherigen GKV-System war immer schon strittig. Unterschiedliche Gerechtigkeitskonzepte (Bedarfsgerechtigkeit vs. Leistungsgerechtigkeit) konkurrieren hier. Allokative Anreizkompatibilitäten werden mikro- wie makroökonomisch herausgestellt. Aber auch die letztendliche Inzidenz steuerfinanzierter Ausgleichszahlungen für soziale Zwecksetzungen in der Gesetzlichen Krankenversicherung bleiben letztendlich fraglich.

Morphologisch verliert die Gesetzliche Krankenversicherung weiterhin an genossenschaftlichem Zuschnitt (Schulz-Nieswandt, 2002). Die Deckung des Bedarfs der Mitglieder auf Gegenseitigkeit wird als Reziprozitätsprinzip innerhalb einer Gesetzlichen Krankenversicherung zugunsten einer undurchsichtigen Finanzierung durch Dritte aufgeweicht.

Schließlich bleibt die europarechtliche Zukunft des GKVSystems unter wettbewerbs-, vergabe- und beihilferechtlichen Regimefragen von der Umstellung auf den Gesundheitsfonds zunächst unberührt. Die Einführung von Wahltarifen und die Umstellung der Kollektivverträge (als Ausdruck der korporatistischen Steuerungsordnung) zugunsten der Selektionsverträge (Einkaufsmodelle der Einzelkassen im Wettbewerb) werden es sein, die durch diesen stillen Marsch in die Quasi-Märkte die Relevanz des Europarechts und der Binnenmarktlogik evozieren werden.

\section{Literatur}

Blonski, Harald (2007). Change Management. Perspektiven, Wege und Aspekte nachhaltiger Veränderung, in Blonski, Harald (Hrsg.), Den Wandel gestalten. Mabuse: Frankfurt. S. 13-72.

Braun, Günter und Jan Güsow (2006). Integrierte Versorgungsstrukturen und Gesundheitsnetzwerke als innovative Ansätze im deutschen Gesundheitswesen, in: Liberalisierung im Gesundheitswesen, hg. von Günther Braun und Frank Schulz-Nieswandt, Baden-Baden, S. 65-93.

Gerlinger, Thomas, Mosebach Kai \& Schmucker Rolf, (2007). Wettbewerbssteuerung in der Gesundheitspolitik, Diskussionspapier 2007-1, Frankfurt am Main. (Internet http://www.kgu.de/zgw/medsoz, Zugriff 27.04.2008).

GKV-WSG (2007). Gesetz zur Stärkung des Wettbewerbs in der gesetzlichen Krankenversicherung (GKV-Wettbewerbsstärkungsgesetz) vom 30. März 2007. BGBl.

Hartweg, Hans-R. (2007). Die Entwicklung der integrierten Versorgung in Deutschland. Berlin: LIT.

Kurscheid, Clarissa (2007). Gründungsmanagement und Netzwerkpflege, in Kurscheid, Clarissa \& Schulz-Nieswandt, Frank (Hg.), Wandel der Versorgungslandschaften im Krankenhaus, Baden-Baden: Nomos, S. 38-49.

Schulz-Nieswandt, Frank (2002). Zur Genossenschaftsartigkeit der Gesetzlichen Krankenversicherung. Weiden-Regensburg: Eurotrans Verlag.

Schulz-Nieswandt, Frank \& Kurscheid, Clarissa (2004). Integrationsversorgung, Münster: Lit-Verlag (2. Auflage i. V.). 